\title{
Solar diameter observations with the Astrolabe at Observatório Nacional - Rio de Janeiro
}

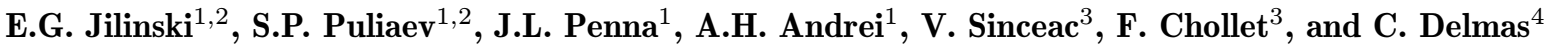 \\ 1 Observatório Nacional/CNPq, Brasil \\ 2 Pulkovo Observatory, Russia \\ 3 DANOF/Observatoire de Paris, France \\ ${ }^{4}$ CERGA/Observatoire de la Côte d'Azur, France
}

Received September 24; accepted November 24, 1997

\begin{abstract}
Observations of the solar diameter are reported from the astrolabe station at Observatório Nacional. All the observations presented here were taken with the 1986CERGA prototype of Variable Prism, which enables many diameter measurements per day and to observe all year round. The 1996 DANOF software for digital CCD image acquisition and data processing has been used. We adopted the recording of $46 \mathrm{CCD}$ frames with direct and reflected images of the limbs along the Sun's transit through the almucantar. The independent observable is the accurate UT datation of each frame.

Here the first campaign results, from January to July/1997, are presented. An asymmetry between the east and west transit is noticeable. The west transit leads to a larger value for the mean semi-diameter and exhibits a larger standard deviation. No clear dependence is found upon the daily results, either on time or on observational parameters. The main outcome of this campaign remains the average solar semi-diameter, from 1149 transits (863 East and 286 West), obtained as 959'.21 \pm 0 ". 03 .
\end{abstract}

Key words: sun: general, fundamental parameters - CCD observations

\section{Introduction}

Determinations of the solar diameter with astrolabes have steadily been pursued by various groups (Laclare 1983; Leister \& Benevides-Soares 1990; Sánchez et al. 1993; Noël 1995; Leister et al. 1996; Golbasi \& Kilic 1997). Comparison of the absolute values from other instruments

Send offprint requests to: E.G. Jilinski, Observatório Nacional/CNPq, R. Gal. José Cristino 77, CEP20921-400, Rio de Janeiro, Brasil. e-mail: jilinski@on.br is difficult, due to unequal definitions of the observational solar limb, to systematic effects peculiar to each technique and, in many instances, to bias introduced by visual observations (Wittmann 1977; Gilliland 1981; Ribes et al. 1988; Débarbat \& Laclare 1990; Laclare et al. 1996). The results presented here are a contribution towards keeping a record of the observed variations of the solar diameter, aimed to add to the astrolabes series and given the low latitude of the station at Rio de Janeiro. The observation of the Sun with astrolabe at the Observatorio Nacional/ CNPq (ON), Rio de Janeiro $\left(\phi=-22^{\circ} 53^{\prime} 42.50^{\prime \prime}, \lambda=+2^{\mathrm{h}} 52^{\mathrm{m}} 53.479^{\mathrm{s}}\right.$, $h=33 \mathrm{~m}$ ) started in 1978 focused on the determination of Earth orbit parameters and on the orientation of the astrometric reference frame (Penna et al. 1996, 1997a). The observations were carried out visually with a $30^{\circ}$ transparent prism and enabled also measurements of the solar diameter. The standard deviation of the daily values for the 1978-1981 campaign was 0.'4 (Penna et al. 1997b), which is comparable with the outcome from other astrolabe stations under the same conditions. Moreover, the average density was greater than 100 observations/year, allowing, thus, to assert the site quality.

In this paper the results from the first seven months campaign, January to July, 1997, are presented. We analyze the results from the point of view of the dependence of several observational parameters, as the observation time of the day and transit length, zenith distance and heliographic latitude, in order to verify whether there are significant effects to be considered when the time series are regarded. The results are discussed in Sect. 3.

All the measurements were taken with a CCD camera for the imaging of the solar limb. The reductions are performed through a constant group of pre-programmed routines upon the digitized images. This ensures a fully "impersonal" result. In the following section the instrument's present method of operation and principles of reduction are briefly described. 
The observations touch the extremes of the Sun's declination and demonstrate the feasibility of the astrolabe observations of the solar diameter all year round, with high density of measurements.

\section{Instrument and data treatment}

The modifications effected upon the Danjon astrolabe at $\mathrm{ON}$ for solar observations were made in the framework of a co-operative project with the CERGA/Observatoire de la Côte d'Azur, aiming at complementary observations (Andrei et al. 1996). They thus followed the design pattern of the CERGA multiple prisms astrolabe (Laclare \& Merlin 1991). The instrument is thermically insulated and a nickel-chrome reflecting $10^{-5}$ filter, peaking on yellow, plate abashes the sunlight. Along the 1.5 hours that a session of measurements in average lasts, the changes of temperature on the instrument did not exceed $4{ }^{\circ} \mathrm{C}$. No influence was found on the intrument's focal distance during one measurement of the solar diameter, which takes from 2 to 7 minutes.

For astrometry, $30^{\circ}$ and $45^{\circ}$ fixed angles reflecting prisms, with zerodur faces are used. The zerodur prisms maintain a very high angular stability in the long term, whereas in the case of the solar diameter measurements a good stability is required just for the few minutes of the Sun's transit through the almucantar. Therefore, for all the observations presented here a front prism formed by two zerodur plates making an adjustable dihedron had been used. The Variable Prism is the 1986 CERGA prototype, working by a system of coils compensation. The functional zenith distances interval so defined extends from about $27^{\circ}$ to $58^{\circ}$.

The observation consists in taking 46 frames of the twin images of the portion of the solar limb crossing the almucantar. Each frame lasts $20 \mathrm{~ms}$ and the observations are taken with regard to the upper and lower limbs. The images are acquired by a COHU \# 4710 CCD camera. The camera is IR sensitive $(4000-10000 \AA)$. The effective wavelength is $6400 \AA$ and the bandpass is $3000 \AA$. Every other vertical line is digitized, producing 256 lines and 512 columns. The vertical pixel size corresponds to 0.5 and thus about $15 \%$ of the solar semi-diameter is imaged.

Following the Observatoire de Paris digital acquisition system software (Sinceac et al. 1997), the solar limb edge, at each CCD line, is represented by the inflection point of the luminosity curve along the line. The observed solar limb can be adequately represented by a parabola that fits the set of inflection points. In each frame, the characteristic parabola's summit admits a horizontal tangent parallel to the CCD columns and is recorded as $(X, Y, t)$, where $X$ and $Y$ are the abscissa and the ordinate while $t$ is the corresponding UTC. The two series of summits, from the direct and reflected twin images, are adjusted by least squares to two crossing lines in the $(Y, t)$ plane. The

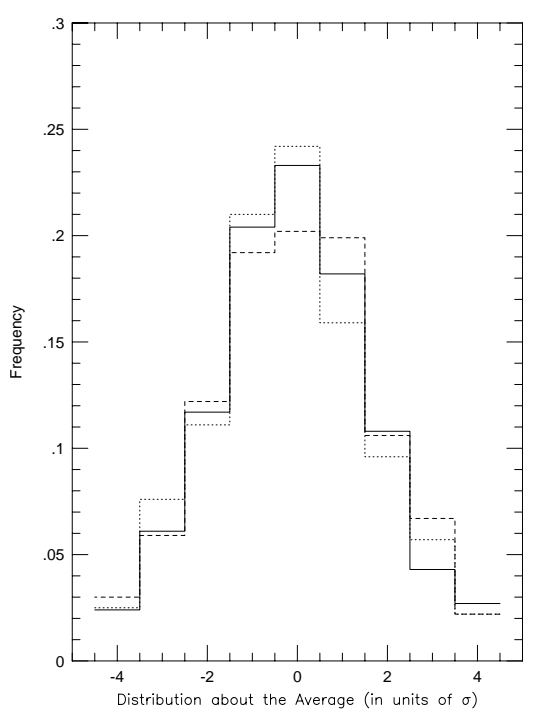

Fig. 1. Frequency distribution of the residuals of the measurements about the average, in units of the standard deviations. The east transits distribution is represented by the dashed line and the west one by the point line. The continuous line traces the histogram for the whole set of observations

location of the point of intersection of these lines gives the instant of crossing of the almucantar by the observed limb. The observation of the crossing of the same almucantar by the second border enables the determination of the Sun's diameter from the difference between the two instants.

\section{Results and discussion}

The results presented here span over 7 months of observations, from January $10^{\text {th }}$ to July $31^{\text {st }}, 1997$. The data thus cover 3 seasons and sizeable ranges in observation length of transit, zenith distance and heliographic latitude, allowing for systematic trends to be searched for. The temperature during the observations varied from about $+42{ }^{\circ} \mathrm{C}$ in summertime (January) down to $+25{ }^{\circ} \mathrm{C}$ in winter (June); being some degrees higher in the afternoons than in the mornings. Figure 1 brings the residuals distribution about the overall semi-diameter average. The distribution shows a distinct normal pattern. We then retain here the measurements under $\pm 3 \sigma$, ascribing the others to observations under adverse meteorological conditions, bad seeing or defective observation. In all, 1149 observations were treated, corresponding to 125 east and west series of measurements of the solar diameter in 91 days. The distribution of the observations appears in Table 1.

The internal errors originate from the definition of the solar limb and the adjusting of the inflection points to a parabola and, then, from the adjusting of the parabolas' summits to crossing lines, from which the time of transit is deduced. Although sharing similar causes, we name external errors those giving a defective superposition of the measured diameters, for an east or west series. In Fig. 2 
Table 1. Number of observations and average solar semidiameter, $\langle\mathrm{SD}\rangle$. From January $10^{\text {th }}$ to July $31^{\text {st }}$, 1997. The "Series" column brings the number of independent daily average semi-diameter values

\begin{tabular}{cccc}
\hline Transit & Measurements & Series & $\langle\mathrm{SD}\rangle(\operatorname{arcsec})$ \\
\hline East & 863 & 91 & $959.14 \pm 0.03$ \\
West & 286 & 34 & $959.41 \pm 0.07$ \\
All & 1149 & 125 & $959.21 \pm 0.03$ \\
\hline
\end{tabular}

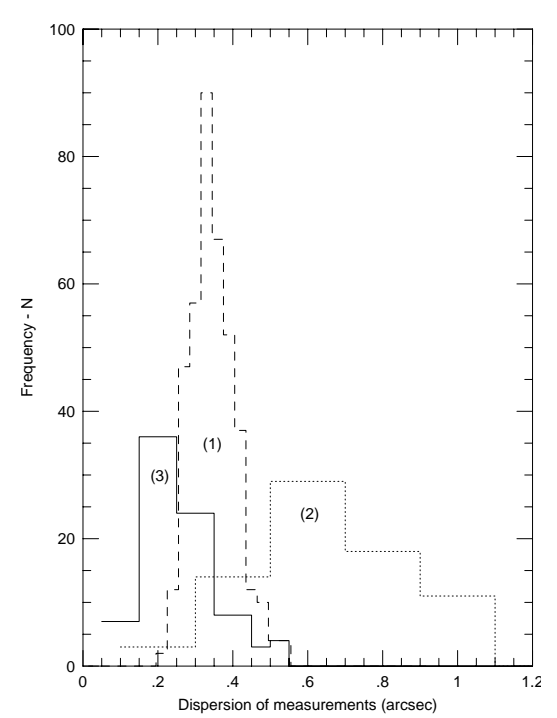

Fig. 2. Distribution of internal and external errors. Errors on the images treatment (1) - for details on the routines we refer the reader to Sinceac (1998); single measurements standard deviations (2) and dispersion of the daily results about the average (3), as given by the number of events $(N)$. All the histograms are relative to east transits only. The west transits show an equivalent, but noisier distribution

the distribution of the internal and external errors is presented. It is seen that the external errors are much larger. Thus the different options of adjustment of the parabolas and even variations on the definition of the solar limb are of minor importance. This supports the approach taken in the present work, in getting as many independent measurements as feasible in a series, to obtain a reliable average.

Figure 3 shows the east and west daily averages. No important trend is clearly apparent, either on the measured semi-diameters or on the errors. In order to verify whether the variation of observational parameters could interfere with the measured semi-diameter, we compared the outcome and the errors of each measurement with the zenith distance, and its trigonometric tangent, and heliographic latitude of transit, as well as with the time length and hour of observation, through a linear least square fit. Also a linear dependence with time was adjusted. The range of the parameters, for the period March-July and the results (angular coefficient) of linear fits of semi-diameters

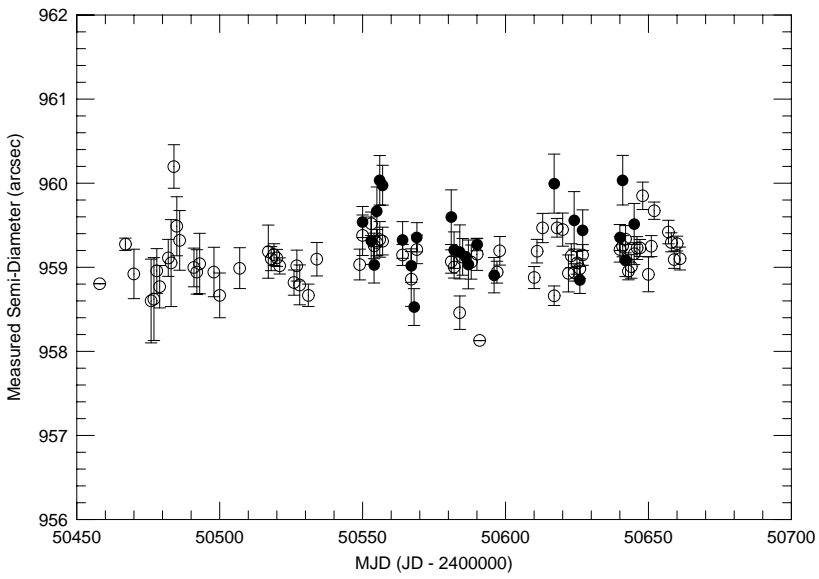

Fig. 3. Daily results. Open circles are east transits and dark circles are west transits

and errors against each of the observational parameters and time are displayed in Table 2. As seen from the table, no important dependence is found, but, perhaps, on $\tan Z$. This result indicates that the relative nature of the measurements renders them unaffected by such variations. In case of the heliographic latitude it must be, however, considered that a longer period of observations would be required, in order to obtain data redundancy and to examine the effect of various degrees of the Sun activity. As for the higher values of the angular coefficient on the $\tan Z$ least squares fit, it indicates that at high zenith distances the agitation of the images can be of importance. The larger values for the west transits support this interpretation. However the errors associated to the so obtained coefficients indicated that such an effect would add up to the series noise, rather than develop a systematic trend. Certainly, with the progress of the project, this aspect can be re-addressed.

Finally, in Table 3 the monthly averages are presented. Again there is no evidence for a trend or a cycle. Thus the only important effect that is apparent in the data set is the difference in the results and precision between the east and west transits, being larger for the later. The afore commented temperature gradient can explain the observed difference. The discrepancy between the averages for the eastern and western transits might be tied with the prototype variable prism observations, as long as this effect was not seen in the Calern series with zerodur fixed angle prisms (Laclare et al. 1996). Nevertheless the smaller number of western observations do not allow a final conclusion, nor to outcast them a priori. So, in spite of the better definition of the eastern transits, all the measurements are kept and the average of the 125 East and West transits is retained. The semi-diameter in this way is found to be $959^{\prime \prime} .21 \pm 0^{\prime \prime} 03$. 
Table 2. Results from the linear fit of the measured solar semi-diameter and the corresponding error against observational parameters. The angular coefficients of the fittings are given for Julian Date (JD), Universal Time of observation, in hours, (UT), Length of the observation, in minutes, $(\Delta T)$, Zenith Distance $(Z)$, Trigonometric Tangent of Zenith Distance (tanZ) and Heliographic Latitude $(b)$



\section{Conclusions}

We presented the results from 1149 measurements of the solar semi-diameter with a modified astrolabe, averaged in 125 east and west series, corresponding to 91 days of observations. East transit results exhibited a larger precision and systematic difference as compared to west ones.

Notwithstanding the experimental character of the 1986 CERGA prototype Variable Prism, used to define the observational zenith distance, the measurements dispersion is comparable to what was obtained with zerodur fixed angle prisms (Laclare 1983). The lower quality measurements in the afternoon, as compared with the morning ones, is of no surprise. It may arises from the temperature differences that can affect the lower layers of the atmosphere and immediate vicinity of the instrument. The stability of the results is a good indication of the variable angle prism performance. A perfectioned prism of this type is currently being developed at OCA/CERGA and will soon replace the prototype used so far.

The digital CCD acquisition methods display a number of advantages over visual observations. It enables an accurate definition of the solar limb, though further modeling of it is in progress. Special care ought to be given to define the wavelength and bandwidth of observation with precision. The method frees the measurement from any personal bias and it helps evaluating the atmospheric turbulence, hence the images quality (Irbah et al. 1994) The method keeps in memory the whole raw data for an eventually better processing. In the case of Observatório Nacional, the acquired images are stored in CD-ROM and DAT tapes.

The site of the Observatório Nacional in Rio de Janeiro comes out as specially fitted for long term surveys of the solar diameter. It characterizes by the great number of
Table 3. Monthly average semi-diameters (in arcseconds). January and February are not included on the table, since the corresponding numbers of observations were small

\begin{tabular}{llll}
\hline Month & East transits & West transits & All transits \\
\hline & & & \\
March & $959.06 \pm 0.04$ & - & $959.06 \pm 0.04$ \\
April & $959.22 \pm 0.06$ & $959.47 \pm 0.08$ & $959.24 \pm 0.05$ \\
May & $959.15 \pm 0.06$ & $959.19 \pm 0.08$ & $959.15 \pm 0.05$ \\
June & $959.08 \pm 0.04$ & $959.53 \pm 0.13$ & $959.13 \pm 0.04$ \\
July & $959.27 \pm 0.03$ & $959.56 \pm 0.09$ & $959.32 \pm 0.03$ \\
\hline
\end{tabular}

possible observations as much as the prospective quality that can be expected, combined with the observation facilities. The seeing of the site is larger than that of Calern, as shown by the averages of similar CCD measurements from this campaign and the 1996-97 Calern's one. The linear dependence between the spreading of the border function and a smaller diameter is confirmed, in line with prior results (Rösch et al. 1983; Neckel 1995).

Since much care is required to define the limb of the solar disk, in order to distinguish between observed diameter variations due to physical shape changes and due to variability in other solar properties affecting the limb definition (Hill \& Stebbins 1975; Hill et al. 1981), further observations are highly desirable of the largest density on time.

Acknowledgements. This work was partially supported by the $\mathrm{CNPq} / \mathrm{CNRS}$, Brazil/ France binational agreement, under the number 910172/95-1. The authors wish also to express their thanks to Francis Laclare (CERGA/OCA), Roberto Vieira Martins, Alberto G.F. dos Santos, the ON Time Service 
Department, Nelson V. Leister (IAG/USP) and Clemence Gneiding (CNPq/LNA) for the contribution to the present research. E.J. and S.P. thank $\mathrm{CNPq}$ for the financial support under the contracts 300016/93-0 and 300017/93-6.

\section{References}

Andrei A.H., Penna J.L., Jilinski E., Puliaev S., 1996, Campaign at VII Reunion Regional Latinoamericana de Astronomia, Resumenes, Uruguai, 1995, Revista Mex. Astron. Astrof., Ser. Conf. 4, 153

Débarbat S., Laclare F., 1990, Acta Astron. 40, 313-319

Gilliland R.L., 1981, AJ 248, 1144-1155

Golbasi O., Kilic H., 1997 (private communication)

Hill H.A., Stebbins R.T., 1975, ApJ 200, 471-483

Hill H.A., Caudell T.P., Bos R.J., 1981, Variations of the Solar Constant, NASA Conf. Pub. 2191

Irbah A., Laclare F., Borgnino J., Merlin G., 1994, Solar Phys. 149,213

Laclare F., 1983, A\&A 125, 200-203

Laclare F., Merlin G., 1991, C. R. Acad. Sci. Paris, t.313, Série II, 323-330

Laclare F., Delmas C., Coin J.P., Irbah A., 1996, Solar Phys. 166,211

Leister N.V., Benevides-Soares P., 1990, C. R. Acad. Sci. Paris, t.311, Série II, 399-404
Leister N.V., Poppe P.C.R., Emilio M., Laclare F., 1996, Dynamics, Ephemerides and Astrometry of the Solar System, S. Ferraz- Mello et al. (eds.), p. 501-504

Neckel H., 1995, Solar Phys. 156, 7-16

Noël F., 1995, A\&AS 113, 131-132

Pecker J-C., 1996, Rev. Mex. AA, (Ser. Conf.) 4, 39-44

Penna J.L., Benevides-Soares P., Andrei A.H., Laclare F., Leister N.V., 1996, A\&A 310, 1036-1042

Penna J.L., Benevides-Soares P., Andrei A.H., Laclare F., 1997a, A\&AS 123, 403-410

Penna J.L., Jilinski E., Puliaev S., Andrei A.H., 1997b, XXIII Reunião anual da SAB, Resumos: Bol. Soc. Astron. Bras., 17, 99

Ribes E., Ribes J.C., Vince I., Merlin P., 1988, C. R. Acad. Sci. Paris, t.307, Série II, 1195-1201

Rösch J., Yerle R., 1983, Solar Phys. 82, 139-150

Sánchez M., Moreno F., Parra F., Soler M., 1993, A\&A 280, 333

Sinceac V., Chollet F., Laclare F., Delmas C., 1997, "Méthode Nouvelle pour la Mesure du Diamètre Solaire avec un Astrolabe" A\&AS (in press)

Sinceac V., 1998, PhD thesis at Paris Observatory. "Analyse et Exploitation des observations du passage du Soleil à partir de l'Astrolabe Danjon équipé d'une Caméra CCD"

Wittmann A., 1977, A\&A 61, 225-227 